\title{
Recording and sorting live human sperm undergoing acrosome reaction
}

\author{
Felipe Carlos Martín Zoppino, Ph.D., Narciso D. Halón, B.S., Matías A. Bustos, M.S., Martín A. Pavarotti, Ph.D., \\ and Luis S. Mayorga, Ph.D. \\ Instituto de Histología y Embriología Dr. Mario H. Burgos (Consejo Nacional de Investigaciones Científicas y Técnicas - Universidad Nacional \\ de Cuyo), School of Medicine, National University of Cuyo, Mendoza, Argentina
}

Objective: To develop and evaluate a method to detect acrosome reaction (AR) in live human sperm.

Design: Prospective study.

Setting: Basic research laboratory.

Patient(s): Human semen samples with normal parameters obtained from healthy donors.

Intervention(s): Acrosome reaction assays.

Main Outome Measure(s): Fluorescence assessment of AR.

Result(s): Evaluating acrosomal exocytosis in live human sperm is challenging. In this study, we report that in reacting sperm, Pisum sativum agglutinin conjugated to fluorescein isothiocyanate rapidly permeates into the acrosome when fusion pores open and stabilizes the acrosomal matrix, preventing the dispersal of the granule contents.

Conclusion(s): Fluorescent Pisum sativum agglutinin can be used to visualize AR in real time, to determine the percentage of sperm undergoing exocytosis upon stimulation, and to separate the population of reacting sperm by flow cytometry. (Fertil Steril ${ }^{\circledR} 2012 ; \square$ : $-\square$. @2012 by American Society for Reproductive Medicine.)

Key Words: Acrosome reaction, human sperm, Pisum sativum agglutinin, flow cytometry, acrosomal matrix

$\mathrm{T}$ he acrosome reaction (AR) is a unique event in the lifespan of sperm characterized by the exocytosis of the acrosomal content and the release of hybrid vesicles formed by patches of the outer acrosomal membrane and the plasma membrane. This complex membrane fusion process exposes the inner acrosomal membrane changing the topology of the plasma membrane and the distribution of proteins that are essential for sperm-egg recognition and fusion (1).

Given the biological importance of the AR, several techniques to assess this exocytic process have been developed (2). Most of them are based on the binding of acrosome-specific probes in fixed cells. Several of these reagents are fluorescent or are coupled to fluorescent dyes. The relatively large size of the acrosomal granule makes it possible to visualize the state of the acrosome of individual spermatozoon by using a standard microscope equipped, when required, with epifluorescence illumination. The manual assessment of the acrosomal state of several hundred of cells is time consuming. Flow cytometry has been used by several groups of investigators to improve acrosomal exocytosis evaluation (3).

Evaluating the acrosome status in live sperm is challenging. A few species have the advantage that the acrosome can be distinguished by phase-contrast and differential interference contrast (DIC) microscopy (2). The AR has been analyzed in real-time in a mouse engineered to express green fluorescent protein (GFP) in the acrosome (4-6). However, for most species the use of

Received January 12, 2012; revised February 14, 2012; accepted March 1, 2012.

F.C.M.Z. has nothing to disclose. N.D.H. has nothing to disclose. M.A.B. has nothing to disclose. M.A.P. has nothing to disclose. L.S.M. has nothing to disclose.

Supported by grants from National University of Cuyo, Argentina (06/J388SeCTyP), Consejo Nacional de Investigaciones Científicas y Técnicas, Argentina (PIP 112-200801-02277), and Agencia Nacional de Promoción Científica y Tecnológica, Argentina (PICT-2008-1114).

Reprint requests: Luis S. Mayorga, Ph.D., Casilla correo 56, 5500 Mendoza, Argentina (E-mail: Imayorga@fcm.uncu.edu.ar).

reagents recognizing the acrosomal content is prevented because the interior of the granule is isolated from the medium by the acrosomal and the plasma membranes. Hence, it is inaccessible for staining unless the cell is fixed and both membranes permeabilized. Interesting to note, at early times during exocytosis, the opening of fusion pores connects the extracellular medium to the acrosomal lumen. The incorporation of two acrosomal markers-soy bean trypsin inhibitor (SBTI) and anti-CD46 antibody-during exocytosis has been used to detect AR in living human sperm (7). The SBTI is a small protein with high affinity for the acrosomal protease acrosin. When added into the incubation medium, SBTI labels the acrosome at the beginning of the exocytosis, when the fusion pores open. Afterward, the SBTI label disappears as the acrosomal content diffuses into the medium. Anti-CD46 is an antibody that specifically recognizes a protein that is located at the inner acrosomal membrane, which is exposed after the dispersion of the acrosomal matrix. When compared to SBTI, it has a slower kinetics of labeling as it requires acrosome content dispersal for binding (7). 
Pisum sativum agglutinin (PSL) is a dimeric lectin, consisting of two $\alpha \beta$ monomers with high affınity for $\alpha$-D-mannose and $\alpha$-D-glucose (8). This lectin conjugated with fluorescein isothiocyanate (PSL-FITC) recognizes several of the highly glycosylated proteins present in the acrosome of several species and stains the acrosomal region of fixed and permeabilized unreacted sperm. After the dispersal of the acrosomal content, the lectin either does not label the acrosome or stains only the equatorial region that retains some acrosomal content after exocytosis. The PSL-FITC has been extensively used to assess AR in fixed human sperm (9). It has also been used to test acrosomal and plasma membrane integrity in unfixed sperm (10). Our aim for this study was to test PSL-FITC as a marker for recording live human sperm undergoing AR.

\section{MATERIALS AND METHODS}

\section{Reagents}

A23187 was from Alomone Labs. Ltd. PSL-FITC and poly-L-lysine were from Sigma-Aldrich Argentina SA. Propidium iodide (PI) and SBTI conjugated to Alexa Fluor 488 (SBTI-Alexa Fluor 488) were from Molecular Probes (Invitrogen Argentina). Bovine serum albumin (BSA) was purchased from ICN (Eurolab SA).

\section{Sperm}

Human semen samples were provided by masturbation from healthy volunteer donors. The informed consent signed by the donors and the protocol for semen sample handling were approved by the Ethic Committee of the School of Medicine, National University of Cuyo. After sample liquefaction (20-30 minutes at $\left.37^{\circ} \mathrm{C}\right)$, highly motile sperm were recovered by swim-up separation for 1.5 hours in human tubal fluid media (HTF; as formulated by Irvine Scientific) supplemented with $5 \mathrm{mg} / \mathrm{mL}$ BSA at $37^{\circ} \mathrm{C}$ in an atmosphere of $5 \% \mathrm{CO}_{2} /$ 95\% air. The sperm suspension was diluted with HTF-BSA to $7 \times 10^{6}$ sperm $/ \mathrm{mL}$ and incubated under the same condition for 3-4 hours to promote sperm capacitation.

\section{Confocal Microscopy}

Human sperm $\left(5 \times 10^{6}\right.$ cells $/ \mathrm{mL}$ in HTF-BSA $)$ were immobilized in poly-L-lysine $(0.01 \%)$ precoated coverslides. Samples were mounted in a chamber at $37^{\circ} \mathrm{C}$ and the medium supplemented with $0.5 \mu \mathrm{g} / \mathrm{mL}$ PI and $5 \mu \mathrm{g} / \mathrm{mL}$ SBTI-Alexa Fluor 488 or $5 \mu \mathrm{g} / \mathrm{mL}$ PSL-FITC. The AR was induced by $10 \mu \mathrm{M}$ of A23187. Images were recorded during 25 minutes in an Olympus FV1000 confocal microscope. Analysis of images was performed using the software Image J (Wayne Rasband, National Institutes of Health).

\section{Epifluorescence Microscopy}

For staining intact acrosomes at the end of an incubation (indirect or classic method of evaluating AR), sperm were washed once with HTF, spotted on slides, air-dried, and fixed/permeabilized in ice-cold methanol for 1 minute. Cells were stained with $25 \mu \mathrm{g} / \mathrm{mL}$ PSL-FITC, $0.5 \mu \mathrm{g} / \mathrm{mL}$ PI in phosphate-buffered saline (PBS) for 40 minutes at room temperature. For staining of acrosomes undergoing AR during incubation (direct method of evaluating AR), $5 \mu \mathrm{g} /$ $\mathrm{mL}$ of PSL-FITC was included in the medium. At the end of the experiment, cells were washed once with HTF and resuspended/fixed in 3\% paraformaldehyde in PBS for 10 minutes at room temperature. Cells were then spotted on slides and air-dried. To stain nuclei, sperm were incubated with 0.5 $\mu \mathrm{g} / \mathrm{mL}$ PI, $1 \%$ saponin in PBS for 15 minutes. Images were recorded in an Olympus FV1000 confocal microscope.

\section{Flow Cytometry}

Sperm $\left(5 \times 10^{6}\right.$ cells $\left./ \mathrm{mL}\right)$ were incubated at $37^{\circ} \mathrm{C}$ in HTF-BSA supplemented with $0.5 \mu \mathrm{g} / \mathrm{mL}$ PI, $5 \mu \mathrm{g} / \mathrm{mL}$ PSL-FITC, and, when required, $10 \mu \mathrm{M}$ A23187. Every 2 minutes, an aliquot of the preparation was analyzed (10,000 events) in a FACSaria III cytometer (Becton Dickinson). The data were analyzed by FACSdiva software (Becton Dickinson).

\section{Transmission Electron Microscopy}

Sperm ( $10^{7}$ cells/condition) were incubated in HTF-BSA supplemented, when required, with $10 \mu \mathrm{M}$ A23187 and/or $5 \mu \mathrm{g} /$ $\mathrm{mL}$ PSL-FITC. After 30 minutes at $37^{\circ} \mathrm{C}$, the samples were fixed in $2.5 \%$ glutaraldehyde/PBS overnight at $4^{\circ} \mathrm{C}$ and prepared for electron microscopy as previously described (11). Ultrathin sections were observed and photographed under a Zeiss 902 electron microscope at $50 \mathrm{kV}$.

\section{RESULTS}

\section{Real Time Recording of AR in Live Human Sperm}

Cells were incubated in the presence of SBTI-Alexa Fluor 488 or PSL-FITC and stimulated with the calcium ionophore A23187. The cells were then analyzed by time-lapse imaging in a confocal microscope. As expected, reacting sperm were transiently stained by SBTI-Alexa Fluor 488 (Fig. 1A and C and Supplemental Video 1, available online). In agreement with the previous study, when acrosomal exocytosis started, SBTI fluorescence showed a rapid accumulation with a peak at 1.5 minutes continued by a progressive loss of fluorescence (7). In the presence of PSL-FITC, a rapid increase of fluorescence was also observed; however, after the initial increase, the fluorescence continued increasing at a slower speed and remained sperm-associated for several minutes (Fig. 1B and $\mathrm{C}$ and Supplemental Video 2, available online). Sperm viability decreased only by $17 \%$ during the 25 minutes of observation as assessed by PI staining of dead cells (Fig. 1D).

\section{PSL-FITC Prevented the Dispersal of Hybrid Vesicles and the Acrosomal Contents}

The observation that PSL-FITC labeling did not decrease over time was unexpected. We decided to analyze the ultrastructure of sperm reacting in the presence of the lectin by transmission electron microscopy (TEM). Human sperm were stimulated with A23187 in the presence or absence of PSL-FITC, fixed, and then prepared for TEM. The lectin did not affect the morphology of unreacted sperm or the percentage of reacted sperm (Fig. 2A and E). However, the morphology of the reacted cells was completely different in sperm incubated with PSL-FITC. Most of 


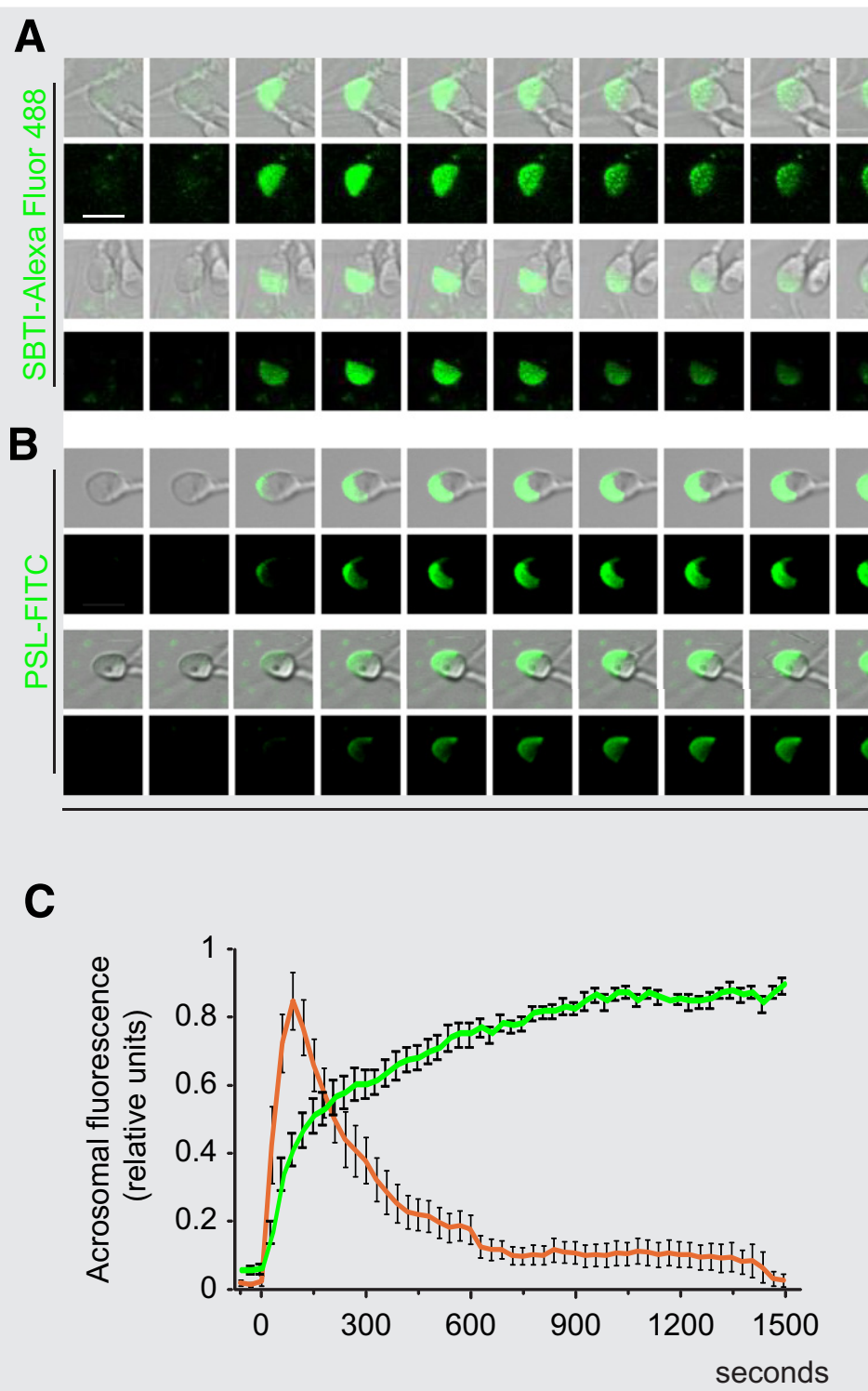

\section{SBTI-Alexa Fluor 488}

D

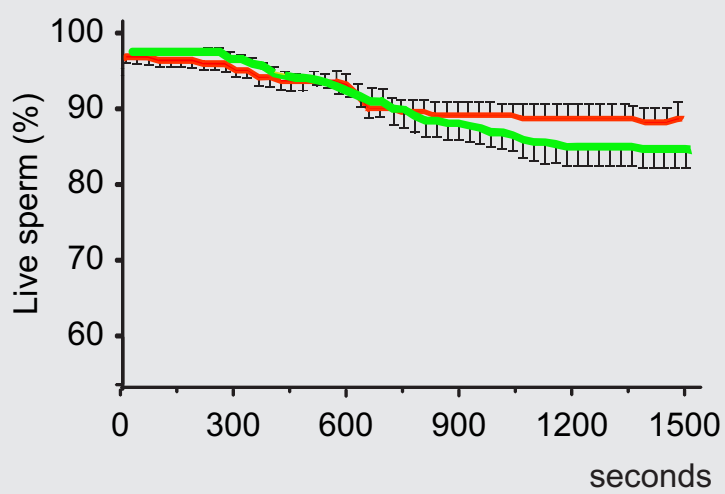

Real-time fluorescence imaging of acrosomal exocytosis in human sperm. Human sperm $\left(5 \times 10^{6} \mathrm{cells} / \mathrm{mL}\right)$ were stimulated with $10 \mu \mathrm{M}$ A23187 in the presence of $0.5 \mu \mathrm{g} / \mathrm{mL}$ propidium iodide and $5 \mu \mathrm{g} / \mathrm{mL} \mathrm{SBTI-Alexa} \mathrm{Fluor} 488$ (A, C, and D) or $5 \mu \mathrm{g} / \mathrm{mL}$ Pisum sativum agglutinin-fluorescein isothiocyanate (PSL-FITC) (B, C, and D), and recorded at 2 frame/minute during 25 minutes in a confocal microscope (A). Sequence of micrographs starting at the time of fusion pore opening in two sperm. Notice that after an initial fluorescence increase, the signal decreased. The top series show the superposition of Alexa Fluor 488 and differential interference contrast microscopy and the bottom series Alexa Fluor 488. Bars $=5 \mu \mathrm{m}$. (B) Same as in A, but in the presence of PSL-FITC. Notice that the fluorescence did not decrease over time. (C) Fluorescence intensity in sperm incubated with SBTI-Alexa Fluor 488 (mean \pm SEM, $N=13$ ) or PSL-FITC (mean \pm SEM, $N=31$ ). The series were synchronized to the time of the initial fluorescence increase in the acrosomal region and normalized to the maximum value recorded. (D) Percentage of live sperm incubated with SBTI-Alexa Fluor 488 or PSL-FITC as assessed by propidium iodide exclusion in three independent experiments (mean \pm SEM).

Zoppino. Labeling acrosome reacting sperm. Fertil Steril 2012.

these cells (about 70\%) were decorated by abundant hybrid vesicles attached to their heads by an electron dense material (Fig. 2C, 2D, and 2F). In contrast, in the absence of PSL-FITC, a very small percentage of the reacted sperm conserved hybrid vesicles (Fig. $2 \mathrm{~B}$ and F). We concluded that the lectin was cross linking the acrosomal content, preventing the diffusion of the material, and tethering the vesicles to the cell. This observation is consistent with the persistent PSL labeling of the acrosome observed by confocal microscopy.

\section{Labeling Sperm Undergoing AR}

The PSL-FITC is used routinely to assess the state of the acrosome in fixed and permeabilized sperm. A sperm with an intact acrosome binds PSL-FITC, whereas a reacted sperm loses 


\section{FIGURE 2}

A

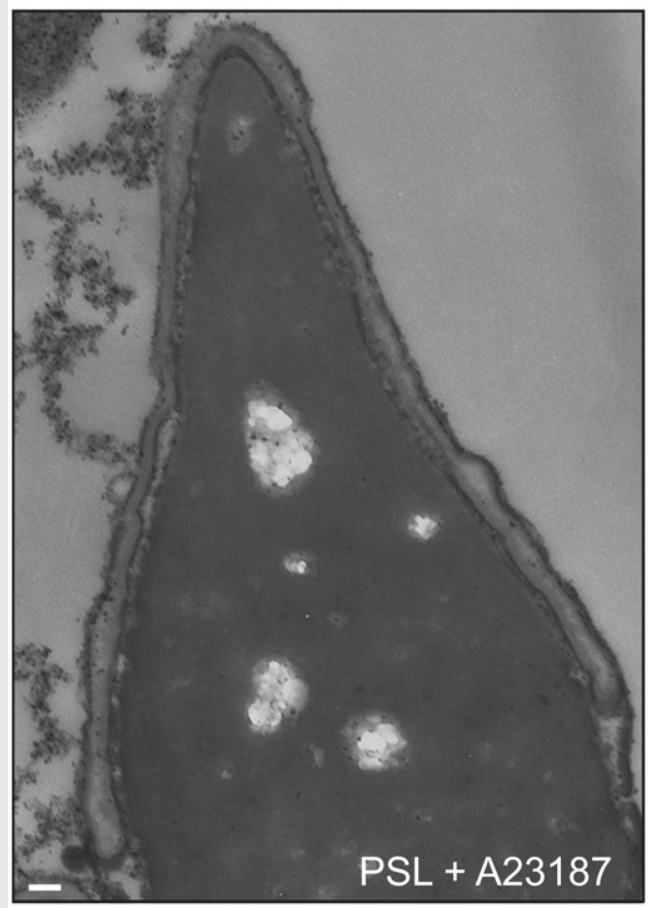

D

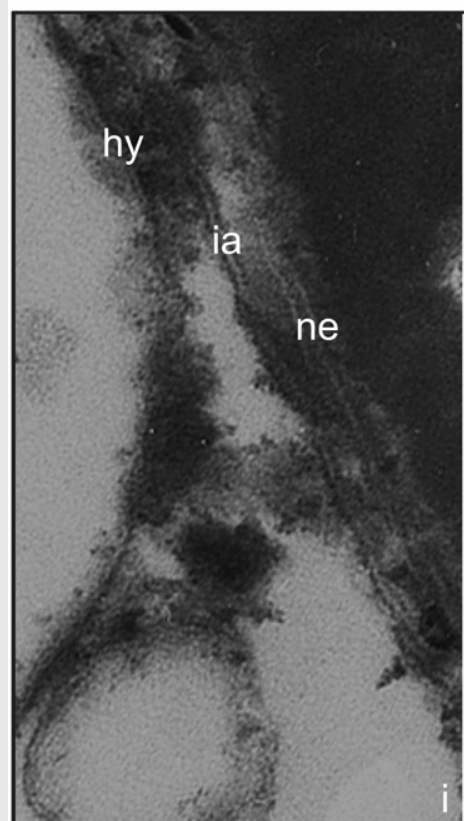

B

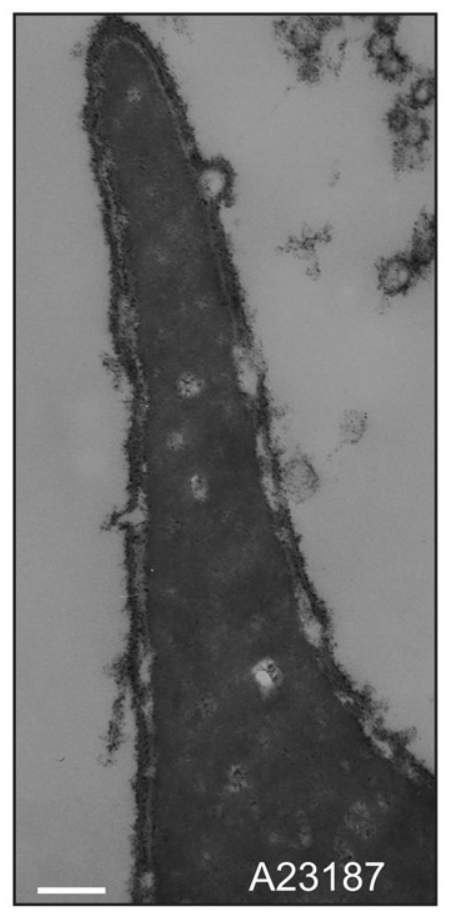

C

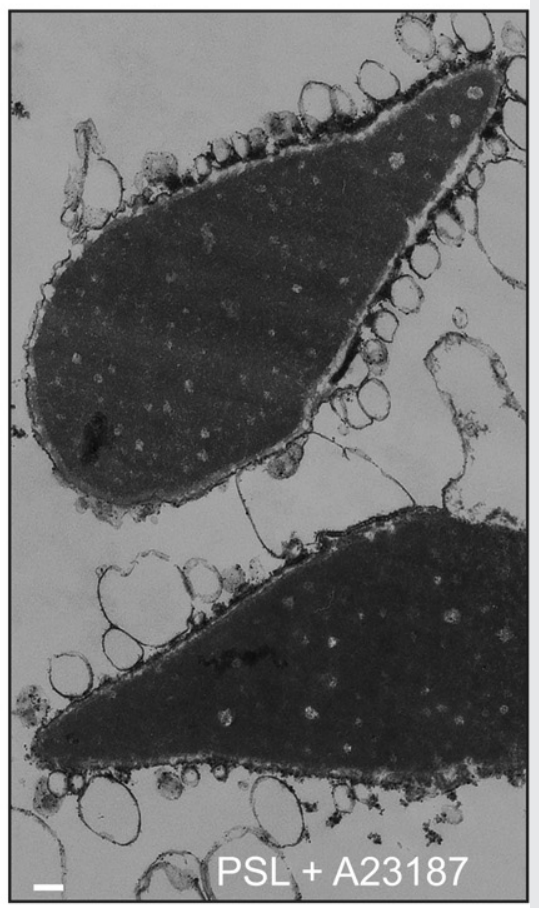

E
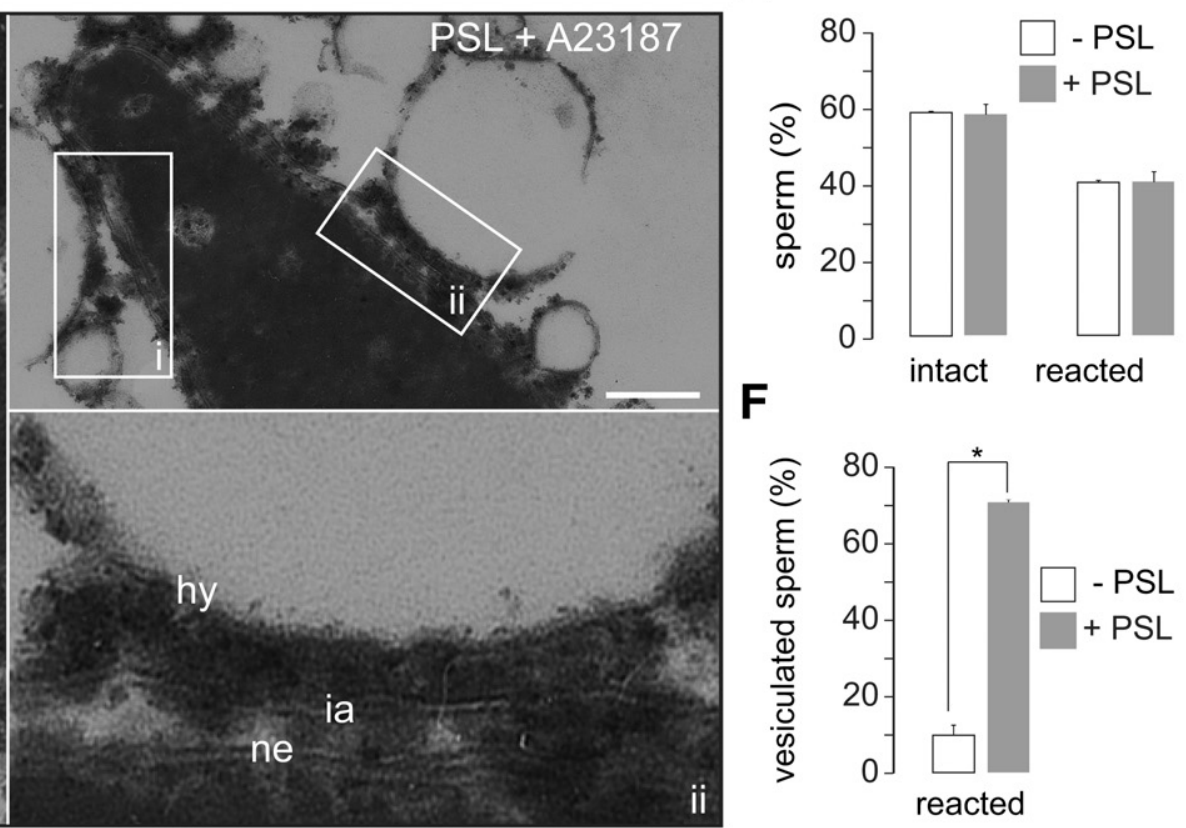

Effect of Pisum sativum agglutinin (PSL) on the ultrastructure of reacting sperm. Sperm ( $10^{7}$ cells/condition) were treated with either $10 \mu \mathrm{M}$ A23187 or A23187 plus $5 \mu \mathrm{g} / \mathrm{mL}$ PSL- fluorescein isothiocyanate (FITC). (A) Sperm with an intact acrosome after incubation with A23187 and PSL-FITC. (B) Reacted sperm after incubation with A23187 in the absence of PSL-FITC. (C) Two highly vesiculated sperm after incubation with A23187 and PSLFITC. (D) Details of a vesiculated sperm treated as in C. Notice the electron dense material tethering the hybrid vesicles (hy) to the inner acrosomal membrane (ia). Bars $=200 \mathrm{~nm}$. Two sectors of this micrograph were enlarged to evidence the membranes and the nuclear envelope (ne). (E) Effect of PSL-FITC on the acrosomal exocytosis (mean \pm range, $N=2$ ). Note that the lectin did not modify the percentage of acrosome reaction. ( $F$ ) Effect of PSL-FITC on the presence of vesicles in reacted sperm (mean \pm range, $N=2$ ). Reacted cells were considered vesiculated when more than five vesicles were attached to the sperm head. A significant increase of vesiculated sperm was observed in sperm treated with PSL-FITC $(P<.05$, Student's t-test for paired observations).

Zoppino. Labeling acrosome reacting sperm. Fertil Steril 2012. 

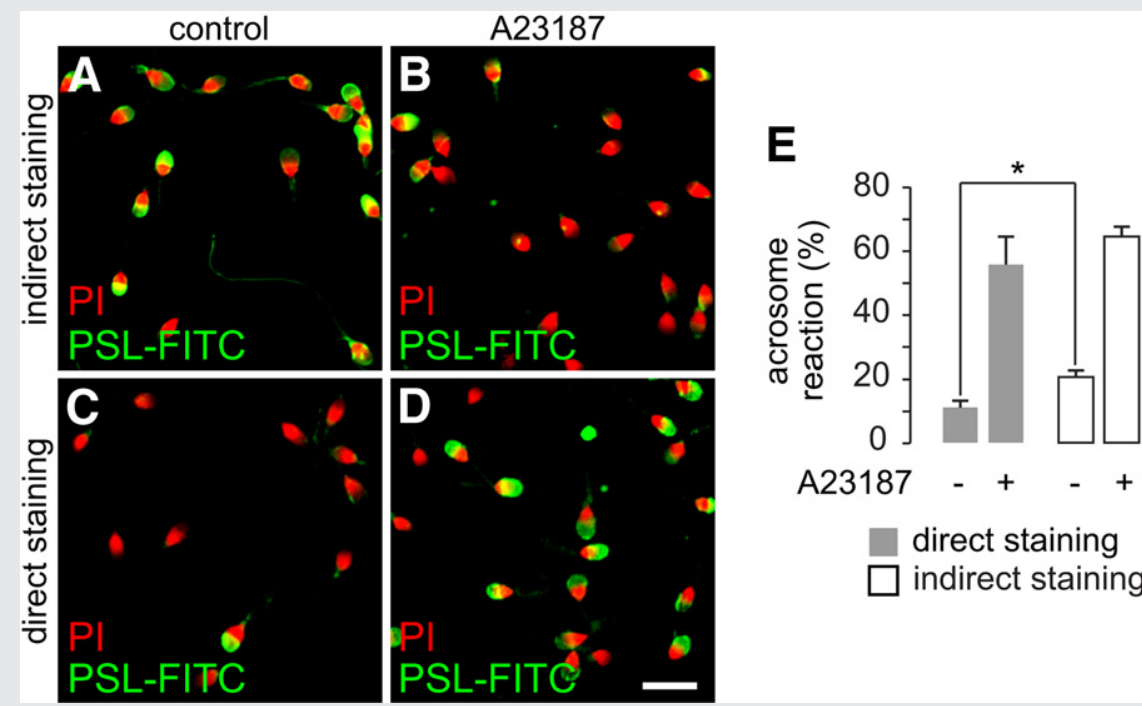

direct staining

indirect staining

Pisum sativum agglutinin-fluorescein isothiocyanate (PSL-FITC) labeling: difference between fresh and fixed/permeabilized sperm. (A-E) Human sperm $\left(7 \times 10^{6}\right.$ cells $\left./ \mathrm{mL}\right)$ were incubated 30 minutes at $37^{\circ} \mathrm{C}$ in human tubal fluid media-bovine serum albumin (HTF-BSA) containing: $\mathrm{A}$, no additions; B, $10 \mu \mathrm{M}$ A23187; C, $5 \mu \mathrm{g} / \mathrm{mL}$ PSL-FITC; D, A23187 + PSL-FITC. Cells were stained by the indirect (classic) protocol (A, B) or the direct method (C, D). Nuclei were labeled after permeabilization with $0.5 \mu \mathrm{g} / \mathrm{mL}$ propidium iodide. Bars $=10 \mu \mathrm{m}$. (E) At least $200 \mathrm{sperm}$ were classified as reacted (nonfluorescent for the indirect method and fluorescent for the direct method) or unreacted (fluorescent for the indirect method and nonfluorescent for the direct method) for each condition. Data represent mean \pm SEM of five independent experiments. * Significantly different $(P<.05$, Student's $t$-test for paired observations).

Zoppino. Labeling acrosome reacting sperm. Fertil Steril 2012.

the lectin-binding material during exocytosis. Testing the percentage of sperm losing PSL staining after an exocytic stimulus (e.g., zona pellucida glycoprotein 3 or progesterone) is an accepted method to measure acrosomal exocytosis $(2,9)$. The fact that PSL-FITC is incorporated during acrosomal exocytosis in live sperm (Fig. 1) indicates that the percentage of sperm undergoing AR during the incubation can be assessed by counting the sperm that become fluorescent during the incubation. A23187-induced exocytosis was tested by the classic (or indirect) method and by including PSL-FITC in the incubation medium before fixation (direct method). As shown in Figure 3A-3D, the patterns of fluorescence were opposite for both techniques; however, the percentage of sperm undergoing AR during the incubation was similar (Fig. 3E). A difference between the two methods is that the direct method only detected sperm reacting during the incubation. Any sperm that had reacted before was not stained by the lectin present in the medium. This is consistent with the lower percentage of reacted cells detected by the direct method in notstimulated sperm (Fig. 3E).

\section{Sorting Live Sperm Undergoing AR by Flow Cytometry}

We decided to take advantage of the observation that live sperm undergoing exocytosis during incubation remain fluorescent for an extended period of time to analyze this population by flow cytometry. The AR was induced by A23187 in the presence of PSL-FITC and PI. Aliquots were taken from the incubation every 2 minutes and analyzed for FITC and PI fluorescence. Some sperm underwent exocytosis as the incubation proceeded and incorporated FITC labeling (Fig. 4A and Supplemental Video 3, available online). Considering FITC and PI labeling, four sperm populations can be distinguished in Figure 4B: unreacted dead sperm (Q1), reacted dead sperm $(\mathrm{Q} 2)$, unreacted live sperm (Q3), and reacted live sperm (Q4). The population of reacted live cells increased steadily up to $60 \%$ after 1 hour of incubation; simultaneously, the number of live cells with intact acrosomes decreased (Fig. 4C). When the experiment was performed at $20^{\circ} \mathrm{C}$ or without A23187, the FITC-labeled population remained very low even after 1 hour of incubation (not shown). Under all conditions, the population of dead sperm was less than $8 \%$ and no significant changes in the cell size or granularity during incubation were observed (not shown). In several experiments, the four populations where sorted by fluorescence-activated cell sorter (FACS) and analyzed by confocal microscopy. Almost 100\% of the cells in the Q4 population (live reacted cells) presented a clear FITC label in the acrosomal region (Fig. 4D, right) showing that the sperm were sorted by acrosome staining and not by unspecific labeling. Meanwhile, the Q3 population did not present FITC labeling (Fig. 4D, left). None were stained by PI, as expected for live sperm. The Q1 and Q2 populations were not analyzed due to the low number of sperm recovered in these fractions.

\section{DISCUSSION}

The sperm AR is a specialized secretory event characterized by profound changes in the topology of the plasma membrane 
FIGURE 4
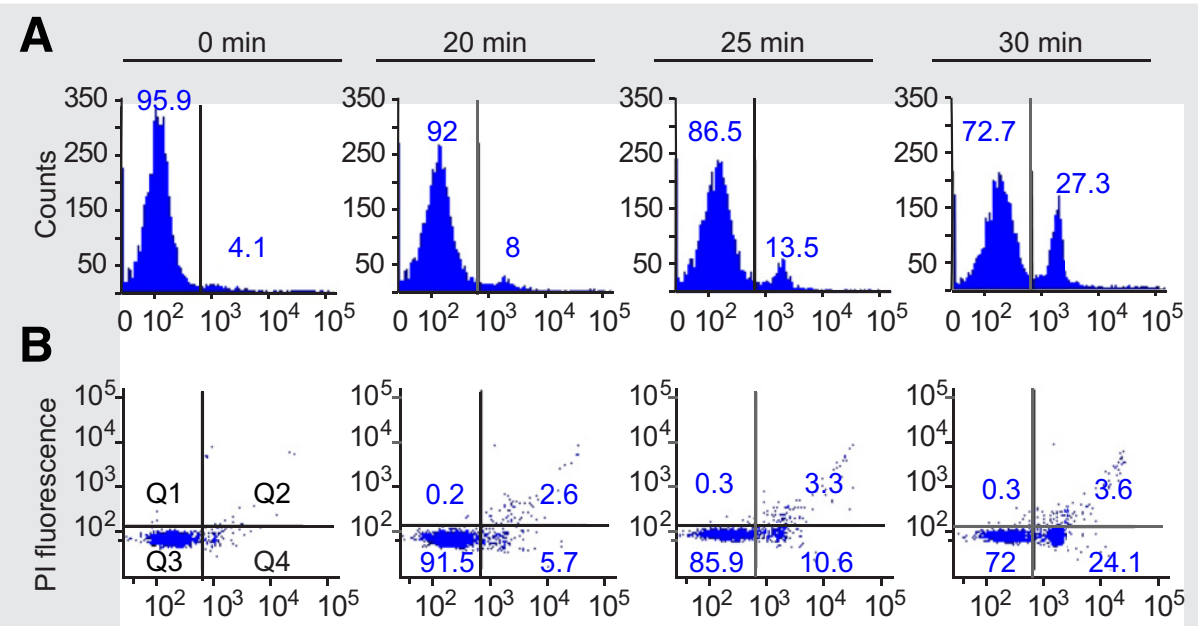

PSL-FITC fluorescence

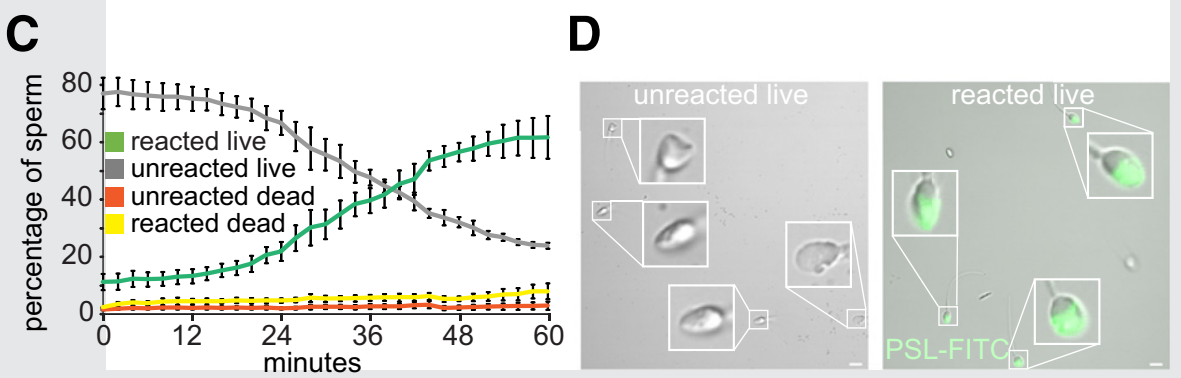

Live reacting sperm populations sorted by fluorescence-activated cell sorter (FACS). Human sperm $\left(5 \times 10^{6}\right.$ cells $/ \mathrm{mL}$ ) were incubated at $37^{\circ} \mathrm{C}$ in human tubal fluid media-bovine serum albumin (HTF-BSA) supplemented with $0.5 \mu \mathrm{g} / \mathrm{mL}$ propidium iodide, $5 \mu \mathrm{g} / \mathrm{mL}$ Pisum sativum agglutininfluorescein isothiocyanate (PSL-FITC), and stimulated with $10 \mu \mathrm{M}$ A23187. Every 2 minutes, 10,000 cells were sorted by FACS according to PSL-FITC and propidium iodide fluorescence. (A) Graphics of a representative experiment showing PSL-FITC fluorescence intensity versus number of PSL-FITC stained cells (counts) over time. Note the sperm population with increased fluorescence that appeared upon incubation. (B) Graphics of PSL-FITC versus propidium iodide fluorescence intensity of the experiment showed in A. The percentage of cells in four populations is indicated: Q1, unreacted dead sperm; Q2, reacted dead sperm; Q3, unreacted live sperm; Q4, reacted live sperm. (C) Percentage of the sperm sorted in experiments as shown in B in Q1-Q4 populations. Data represent mean \pm SEM of three independent experiments. (D) Confocal images of sperm sorted by FACS correspond to Q3 (left) and Q4 (right). Note that PSL-FITC localized to the acrosome region the sperm in Q4 and that propidium iodide staining was negative in Q3 and Q4. Bars $=10 \mu \mathrm{m}$. Insets show details of the images at a larger magnification.

Zoppino. Labeling acrosome reacting sperm. Fertil Steril 2012.

and the distribution of proteins that are essential for spermegg recognition and fusion $(1,12)$. This dynamic process has been analyzed in detail for the human sperm at different times after stimulation in fixed cells (13). At present, many groups of investigators are analyzing in real time the physicochemical changes occurring in these cells upon stimulation. Still, there are very few reports showing $\mathrm{AR}$ in human sperm in real time experiments. This was achieved in an elegant work where SBTI and anti-CD46 antibody were used to detect AR (7). The SBTI is rapidly incorporated into the acrosome as the fusion pores open, and rapidly leaves the acrosome as the granule content is dispersed. The antibody, which recognizes an antigen in the inner acrosomal membrane, follows a completely different pattern. It only stains sperm after the dispersal of the acrosomal matrix. The PSL follows incorporation kinetics similar to SBTI, but the label is not lost for an extended time. We showed by electron microscopy that in the presence of the lectin, the hybrid vesicles formed during exocytosis are retained. Each molecule of PSL is a dimer capable of binding simultaneously two carbohydrate residues (14). This property is probably responsible for stabilizing the acrosomal matrix preventing the dispersal of the acrosomal content and tethering the vesicles to the sperm head. The PSL stable labeling is in fact a useful characteristic that we have exploited to evaluate acrosomal exocytosis in different experimental protocols.

By confocal microscopy, the increase in fluorescence upon opening of fusion pores connecting the extracellular medium with the acrosomal lumen is easy to detect. It will be interesting to follow the AR using high resolution microscopy that will provide spatial and time-related details about the vesiculation process. Analyses of the secretory process in real time is at present essential to correlate the large body of evidence showing dynamic changes occurring in the sperm upon stimulation (e.g., changes in ion concentrations, $\mathrm{pH}$, membrane polarization) with the exocytic process. 
The inclusion of lectin during stimulation is also a useful variation to evaluate AR in fixed cells. It is simple and has the advantage of reducing the background of spontaneously reacted sperm detected by the classic postfixation staining method. In this experimental condition, only sperm reacting during the incubation incorporate the lectin. The label resists perfectly the fixation procedure, rendering brilliant cells with well-defined acrosomal staining. Sperm reacted before the incubation were not labeled by the lectin or presented an equatorial stain.

The fact that live reacted sperm can be sorted from the rest of the cells by FACS has several potentially important implications. For basic studies, the biochemical and functional differences between reactive and nonreactive cells will be of outstanding importance. At present, it is an unsolved issue that only a limited percentage of human sperm respond to stimulation. The possibility of recovering live reacted sperm may also be important for improving IVF procedures. Several reports indicate that reacted sperm have better performance for intracytoplasmic sperm injection (ICSI) $(15,16)$. In addition, the acrosome itself may have deleterious effects for reproduction (17). It is possible that PSL-stained sperm will not be suitable for direct use in IVF procedures and additional treatments will be required to eliminate the lectin. In conclusion, in this report we describe innovative methods for analyzing $A R$ in live human sperm that open up a range of possibilities for studying this complex exocytic process.

Acknowledgments: The authors thank Graciela Gutierrez, Alejandra Medero, Cristina Aguilera, Marcelo Furlan, Florencia Gatti, and Andrea Lafalla for excellent technical assistance and Claudia Tomes and Silvia Belmonte for critically reading the manuscript.

\section{REFERENCES}

1. Inoue $\mathrm{N}$, Ikawa M, Okabe M. The mechanism of sperm-egg interaction and the involvement of IZUMO1 in fusion. Asian J Androl 2011;13:81-7.

2. Cross NL, Meizel S. Methods for evaluating the acrosomal status of mammalian sperm. Biol Reprod 1989;41:635-41.
3. Hossain MS, Johannisson A, Wallgren M, Nagy S, Siqueira AP, RodriguezMartinez H. Flow cytometry for the assessment of animal sperm integrity and functionality: state of the art. Asian J Androl 2011;13:406-19.

4. Nakanishi T, Ikawa M, Yamada S, Parvinen M, Baba T, Nishimune $Y$, Okabe M. Real-time observation of acrosomal dispersal from mouse sperm using GFP as a marker protein. FEBS Lett 1999;449:277-83.

5. Jin M, Fujiwara E, Kakiuchi Y, Okabe M, Satouh Y, Baba SA, Chiba K, Hirohashi N. Most fertilizing mouse spermatozoa begin their acrosome reaction before contact with the zona pellucida during in vitro fertilization. Proc Natl Acad Sci U S A 2011;108:4892-6.

6. Buffone MG, Rodriguez-Miranda E, Storey BT, Gerton GL. Acrosomal exocytosis of mouse sperm progresses in a consistent direction in response to zona pellucida. J Cell Physiol 2009;220:611-20.

7. Harper CV, Cummerson JA, White MR, Publicover SJ, Johnson PM. Dynamic resolution of acrosomal exocytosis in human sperm. J Cell Sci 2008;121: 2130-5.

8. Einspahr H, Parks EH, Suguna K, Subramanian E, Suddath FL. The crystal structure of pea lectin at 3.0-A resolution. J Biol Chem 1986;261:16518-27.

9. Mendoza C, Carreras A, Moos J, Tesarik J. Distinction between true acrosome reaction and degenerative acrosome loss by a one-step staining method using Pisum sativum agglutinin. J Reprod Fertil 1992;95:755-63.

10. Farlin ME, Jasko DJ, Graham JK, Squires EL. Assessment of Pisum sativum agglutinin in identifying acrosomal damage in stallion spermatozoa. Mol Reprod Dev 1992;32:23-7.

11. Zanetti N, Mayorga LS. Acrosomal swelling and membrane docking are required for hybrid vesicle formation during the human sperm acrosome reaction. Biol Reprod 2009;81:396-405.

12. Yanagimachi R. Mammalian fertilization. In: Knobil E, Neill JD, editors. The physiology of reproduction. New York: Raven Press; 1994:189-281.

13. Nagae $T$, Yanagimachi R, Srivastava PN, Yanagimachi H. Acrosome reaction in human spermatozoa. Fertil Steril 1986;45:701-7.

14. Roy R, Page D, Perez SF, Bencomo W. Effect of shape, size, and valency of multivalent mannosides on their binding properties to phytohemagglutinins. Glycoconj J 1998;15:251-63.

15. Gianaroli L, Magli MC, Ferraretti AP, Crippa A, Lappi M, Capitani S, Baccetti B. Birefringence characteristics in sperm heads allow for the selection of reacted spermatozoa for intracytoplasmic sperm injection. Fertil Steril 2010;93:807-13.

16. Morozumi K, Shikano T, Miyazaki S, Yanagimachi R. Simultaneous removal of sperm plasma membrane and acrosome before intracytoplasmic sperm injection improves oocyte activation/embryonic development. Proc Natl Acad Sci U S A 2006;103:17661-6.

17. Morozumi K, Yanagimachi R. Incorporation of the acrosome into the oocyte during intracytoplasmic sperm injection could be potentially hazardous to embryo development. Proc Natl Acad Sci U S A 2005;102:14209-14. 
Supplemental Video 1. Real-time observation of acrosomal exocytosis in human sperm by SBTI-Alexa 488 labeling. Human sperm $\left(5 \times 10^{6}\right.$ cell $\left./ \mathrm{mL}\right)$ were stimulated with $10 \mu \mathrm{M}$ A23187 in the presence of $5 \mu \mathrm{g} / \mathrm{mL}$ SBTI-Alexa Fluo 488 (to label acrosome in reacted sperm) and $0.5 \mu \mathrm{g} / \mathrm{mL}$ propidium iodide (used as dead sperm marker). The movie was edited with a section of confocal images ( $\times 60$ objective) taken every 30 seconds during 25 minutes. Acrosome reaction is denoted by a green fluorescence localized in the acrosome region. Dead cells are red stained with propidium iodide.

Supplemental Video 2. Real-time observation of acrosomal exocytosis in human sperm by Pisum sativum agglutininfluorescein isothiocyanate (PSL-FITC) labeling. Human sperm $\left(5 \times 10^{6}\right.$ cell $\left./ \mathrm{mL}\right)$ were stimulated with $10 \mu \mathrm{M}$ A23187 in the presence of $5 \mu \mathrm{g} / \mathrm{mL}$ PSL-FITC (to label acrosome in reacted sperm) and $0.5 \mu \mathrm{g} / \mathrm{mL}$ propidium iodide (used as dead sperm marker). The movie was edited with a section of confocal images ( $\times 60$ objective) taken every 30 seconds during 25 minutes. Acrosome reaction is denoted by a green fluorescence localized in the acrosome region. Dead cells are red stained with propidium iodide.
Supplemental Video 3. Analysis of human acrosome reaction by Pisum sativum agglutinin-fluorescence-activated cell sorter (PSL-FACS). Human sperm $\left(5 \times 10^{6}\right.$ cell $\left./ \mathrm{mL}\right)$ were stimulated with $10 \mu \mathrm{M}$ A23187 and incubated at $37^{\circ} \mathrm{C}$ in the presence of $5 \mu \mathrm{g} / \mathrm{mL}$ PSL-fluorescein isothiocyanate (FITC) and $0.5 \mu \mathrm{g} / \mathrm{mL}$ propidium iodide. Every 2 minutes (for 1 hour), 10,000 cells were sorted by fluorescence-activated cell sorter according to PSL-FITC and propidium iodide fluorescence. The movie shows animated graphics of PSL-FITC fluorescence intensity versus number of PSL-FITC stained cells (counts) and PSL-FITC versus propidium iodide fluorescence intensity. The areas in the video correspond to $\mathrm{P} 4$ reacted sperm; $\mathrm{P} 5$ unreacted sperm; Q1, unreacted dead sperm; Q2, reacted dead sperm; Q3, unreacted live sperm; Q4, reacted live sperm. 